\title{
Mehrsprachigkeit als Verfahren der gesellschaftlichen Inklusion in schwedischer Gegenwartsliteratur am Beispiel von Neftali Milfuegos' Tankar mellan hjärtslag (2015) und Sami Saids Väldigt sällan fin (2012)
}

\begin{abstract}
Der Artikel verfolgt eine Tendenz in der schwedischsprachigen Gegenwartsliteratur, in der gesellschaftliche Inklusion wesentlich durch Spracharbeit vorgeführt wird. Anhand zweier Beispiele zeigt er Strategien auf, durch die mehrsprachige Literatur ihre multinationalen Protagonisten in einer schwedischen Gesellschaft verankert, deren monolinguale und -kulturelle Vorstellung gleichzeitig unterlaufen wird. Damit werden sprachbasierte Dichotomien des ,Wir‘ und der ,Anderen', wie sie auch der Annahme unterliegen, dass literarische Mehrsprachigkeit zum Beispiel die Funktion erfülle, Minderheitensprachen sichtbar zu machen, für nichtig erklärt.
\end{abstract}

Keywords: Mehrsprachigkeit; gesellschaftliche Inklusion; schwedische Gegenwartsliteratur; code-switching; Neftali Milfuegos; Sami Said; Tigrinisch

Der implizit oder explizit auftretenden Mehrsprachigkeit in literarischen Texten werden neben persönlichen oder ästhetischen Gründen diverse Funktionen zugeschrieben. ${ }^{1}$ Die schwedische Linguistin Carla Jonsson, zum Beispiel, beschreibt literarische Mehrsprachigkeit in Bezug auf das Verhältnis der offiziellen schwedischen Minderheitensprachen Sami und Meänkieli zum Standardschwedischen in dem Jugendbuch Sms från Soppero (2007, SMS aus Soppero) von Ann-Helén Laestadius und dem Roman Populärmusik från Vittula (2000, Populärmusik aus Vittula) von Mikael Niemi als eine Art Machtinstrument. In dieser Form könne Mehrsprachigkeit in Literatur unter anderem auf unterdrückte Stimmen aufmerksam machen und monolinguale Diskurse herausfordern:

The use of code-switching is inextricably linked to the concept of power. Code-switching can be used to resist, challenge and transform power relations and domination, to make silent

1 Vgl. für eine ausführlichere Aufzählung Laakso 2011, 28-34.

2 Open Access. (C) 2021 Hannah Tischmann, published by De Gruyter. (c) BY-NC-ND This work is licensed under the Creative Commons Attribution-NonCommercial-NoDerivatives 4.0 International License. 
voices heard, to legitimize a certain linguistic variety (eg. [sic!] minority languages, multiethnic varieties) [...] and to legitimize the very use of code-switching, to challenge dominant notions of language alternation as being deviant, to create an alternative marketplace for multilingual literary texts, to empower minority groups, to decenter the ,legitimate language (eg. [sic!]) and to promote heteroglossia and resist monologism. (Jonsson 2012, 226)

Laut Jonsson (2012, 223-224) erfolgt eine solche Legitimierung und Sichtbarmachung der Minderheitensprachen und ihrer Sprecher nicht zuletzt durch den Effekt einer Entfremdung auf Seiten der Leser, wenn diese selbst durch ihre fehlenden Sprachkenntnisse in eine externe Position gedrängt werden.

Johanna Laakso $(2011,33)$ verweist auf eine weitere mögliche Funktion literarischer Mehrsprachigkeit: Durch einen humoristischen Effekt sowie den Bezug auf regionale Identitäten könne sie charakterspezifische Eigenschaften der Figuren hervorheben, die in diesem Fall allerdings typischerweise Tölpelhaftigkeit und Bildungsmangel darstellen und die Figuren lächerlich machen würden.

Die Funktionszuschreibungen und Effekte von Mehrsprachigkeit in literarischen Texten, wie sie von Jonsson und Laakso beschrieben werden, beruhen wesentlich darauf, dass der Wechsel zwischen Sprachen oder Sprachvarietäten eine Dichotomie zwischen dem Eigenen und dem konstruierten ,Anderen' hervorruft. Entweder werden die ,Anderen` als Sprecher einer Minderheitensprache aus ihrer unterlegenen Position herausgehoben, beziehungsweise diejenigen, die die Minderheitensprache nicht sprechen, als die,Anderen' konstruiert, oder Individuen werden durch ihre individuelle Sprachfärbung als ,anders ' - und dabei nicht selten als unterlegen - betrachtet.

Solch sprachbasierte, vorschnelle Unterscheidungen von Individuen, die sich geographisch betrachtet an einem gemeinsamen Ort befinden, scheinen nicht ungewöhnlich. Die Wahrnehmung von Dichotomien und die Diskriminierung aufgrund sprachlicher Vielfalt ist jedoch höchst erstaunlich und alarmierend, wenn man bedenkt, dass wir uns im globalen Zeitalter befinden, in dem internationale Erfahrungen sowie Sprachkompetenzen eigentlich als wichtige Schlüsselqualifikationen angesehen werden. Monokulturelle Nationen werden zwar zunehmend von rechten politischen Gruppierungen angestrebt, entsprechen aber keineswegs dem Streben nach Diversität und Toleranz, wie es zum Beispiel das Motto der Europäischen Union „In Vielfalt geeint“ impliziert.

In der schwedischen Literatur sind die Wahrnehmung und die Grundlosigkeit stereotyper Charakterisierungen in den vergangenen Jahren zunehmend herausgefordert worden, indem Mehrsprachigkeit als symptomatisches Element des ,Anderen' dekonstruiert wird. Solche Verfahren weisen unter anderem die Debütromane Tankar mellan hjärtslag (2015, Gedanken zwischen Herzschlägen) von Neftali Milfuegos und Väldigt sällan fin (2012, Sehr selten schön) von Sami 
Said auf. Im Folgenden wird an ausgewählten Aspekten gezeigt, wie sie durch ihren inhaltlichen und stilistischen Gebrauch von Mehrsprachigkeit die Dichotomie des Fremden und des Eigenen aufbrechen, anstatt sie durch die Einführung , anderer' Elemente zu konstruieren. Mononationale Identitäten werden dementsprechend hinterfragt, stattdessen wird ein multikulturelles, multilinguales und religiös vielfältiges Schweden vorgeschlagen. Literaturhistorisch schreiben sich die Romane damit in eine noch junge Tradition ein, die sich in Schweden zu Beginn des 21. Jahrhunderts mit Texten wie Jonas Hassen Khemiris Ett öga rött (2003, Das Kamel ohne Höcker) formiert. Gleichzeitig entwickeln sie diese jedoch weiter, indem sie zum Beispiel geographische Aspekte als Determinanten sprachlicher, kultureller und nationaler Zugehörigkeiten in Frage stellen, wie sich in der abschließenden Diskussion von literarischer Mehrsprachigkeit als Verfahren der Inklusion zeigen wird.

Dabei dient die linguistische Terminologie, die in den folgenden Ausführungen auf die literarischen Texte angewendet wird, unter Berücksichtigung der literaturspezifischen Möglichkeiten bei der Verhandlung und dem Entwurf von Mehrsprachigkeit als Unterstützung der literaturwissenschaftlichen Perspektive. Es handelt sich bei den Untersuchungen also um keine vordergründig sprachwissenschaftlichen Analysen, die auf empirische Erkenntnisse außerhalb der fiktiven Welt zielen.

Die verwendeten linguistischen Begriffe beziehen sich insbesondere auf die verschiedenen Formen von Mehrsprachigkeit. Interlinguales code-switching wird als Sprachwechsel zwischen verschiedenen Sprachen definiert, während intralinguales code-switching den Wechsel zwischen Sprachvarietäten beschreibt. (Vgl. Landqvist 2012, 13) Beide Varianten können im literarischen Text implizit, d. h. durch den Kontext oder durch Metakommentare signalisiert, oder explizit, beispielsweise in Form von Entlehnungen oder tatsächlichem code-switching in der Erzähler- und/oder Figurenrede, auftreten. ${ }^{2}$ (Vgl. Eriksson und Haapamäki 2011, 45-46)

2 Zum Begriff des code-switching als Sprachwechsel zwischen Sprachen und Sprachvarietäten vgl. Landqvist 2014, 60. 


\section{Widerstand und Zugehörigkeit in Neftali Milfuegos' Tankar mellan hjärtslag und Sami Saids Väldigt sällan fin}

Neftali, die Hauptfigur in Neftali Milfuegos’ Roman Tankar mellan hjärtslag ${ }^{3}$, ist in Södermalm, einem hippen Stockholmer Stadtteil, aufgewachsen. Aufgrund seiner chilenischen Wurzeln väterlicherseits fühlt er sich jedoch ständig ausgegrenzt. Mehr und mehr verachtet Neftali schwedischen Rassismus sowie nationalistische und kapitalistische Bestrebungen im Allgemeinen. Als er mit seinem Journalismuskurs nach Chile fährt, will er sich von allem, was er als typisch schwedisch empfindet, loslösen. Dieses gelingt ihm allerdings nur bedingt. Die schwedische Sprache ist ständig präsent und ein ,richtiger' Chilene scheint er mit seiner schwedischen Sozialisierung ebenfalls nicht zu sein.

Ebenso wie Neftali versucht auch Noha, die Hauptfigur in Sami Saids Väldigt sällan fin, sich von seiner schwedischen Lebenswelt $\mathrm{zu}$ isolieren, indem er seine streng muslimische Erziehung, die er eigentlich kritisiert, als unvereinbar mit einem Studentenleben in Linköping ansieht. Nohas Konstruktion des ausgeschlossenen Fremden wird jedoch im Laufe des Romans brüchig. Zwei Schweden, die sich von seiner abweisenden Haltung nicht beeindrucken lassen, fordern ihn durch ihre Loyalität dazu heraus, sein soziales Verhalten und seine Position in der schwedischen Gesellschaft zu überdenken. Diese Veränderung geht einher mit einem Besuch in Nohas Geburtsland Eritrea nach dem Tod des Großvaters und dem Zugeständnis seines Vaters, dass religiöse Prinzipien von Generation zu Generation eine andere Bedeutung bekommen können.

Nohas und Neftalis Sprachgebrauch und die Art, wie Mehrsprachigkeit auch auf der discours-Ebene der Romane auftritt, suggerieren auf vielfältige Weise eine als selbstverständlich auftretende Zugehörigkeit dieser multikulturell aufgewachsenen Hauptfiguren zur schwedischen Gesellschaft und tragen zur Konstruktion ihrer Identitäten bei. Laut dem Anthropologen Richard Bauman ist Identität nämlich die

emergent construction, the situated outcome of a rhetorical and interpretive process in which interactants make situationally motivated selections from socially constituted repertoires of identificational and affiliational resources and craft these semiotic resources into identity claims for presentation to others. (Bauman 2000,1)

3 Aufgrund der Namensgleichheit wird die Romanfigur im Folgenden mit „Neftali“ bezeichnet, während sich „Neftali Milfuegos“ auf den Autor bezieht. 
Somit basiert Identität wesentlich auf linguistischen Merkmalen wie dem Sprachstil, der nach Asif Agha (2007, 81) durch enregisterment zu eigenen und fremden sozialen Positionsbestimmungen dienen kann. Dieses wird insbesondere in Tankar mellan hjärtslag deutlich, wenn Neftali seinen Sprachstil bewusst an stereotype Erwartungen seines Gegenübers anpasst und diese folglich als Illusion entblößt:

\begin{abstract}
Jag mindes de gånger folk försökt snacka med mej på vad de upplevt som mitt språk. - Ey yo yo mannish, shu bräää, va händish lään. Med en kefft härmad brytning och viftande armar som en MC. [...] Jag mindes alla sammanhangen där jag förväntades fylla en roll. Och jag fyllde den. De trodde sej veta vem jag var utifrån mitt tugg å utseende. Uppfattades som drivarn, flipparn, den oseriöse. Blatten. (Det här var bara bland svenskar.) Å jag tyckte det var kul att driva med deras stereotypa bild. Leka hård förortskis fast jag kom från mer prioriterade områden. Många gånger hade detta etsat sej fast hos åskådarna så till den grad att leken format deras bild av mej. Men det gick att bryta. Det räckte med att jag delade med mej av reflekterande tankar. (Milfuegos 2015, 29-30)
\end{abstract}

[Ich erinnerte mich an die Male, als Leute versucht haben, mit mir auf der Sprache zu sprechen, die sie als die meine wahrnahmen. - Ey yo yo, Bruder, was geht Alda? Mit einem schlecht nachgemachten Akzent und fuchtelnden Armen wie ein MC. [...] Ich dachte an alle Zusammenhänge, bei denen von mir erwartet wurde, eine Rolle zu erfüllen. Und ich erfüllte sie. Sie glaubten, ausgehend von meiner Sprache und meines Aussehens zu wissen, wer ich war. Wurde als der Herumtreibende, der Freak, der Unseriöse gesehen. Der Kanake. (Das passierte nur unter Schweden.) Und ich fand es lustig, ihr stereotypes Bild zu verarschen. Den harten Vorortburschen zu spielen, obwohl ich aus einer besseren Gegend kam. Viele Male hatte sich dieses in den Zuschauern bis zu dem Grad festgeätzt, dass das Spiel ihr Bild von mir geformt hatte. Aber man konnte es durchbrechen. Es reichte, wenn ich reflektierte Gedanken von mir gab. $]^{4}$

Neftalis Sprachgebrauch ist durch eine Varianz an schwedischen Dialekten und Soziolekten geprägt, die zusammen mit spanischen Ausdrücken das sogenannte Standardschwedisch durchziehen. Dieses explizit und implizit auftretende interund intralinguale code-switching führt zum einen dazu, dass spanische Ausdrücke bei fehlender Kursivierung zu einem natürlichen integrativen Teil von Neftalis schwedischem Sprachstil werden. Diese stehen nämlich im Gegensatz zu kursivierten Begriffen, die durch ihre typografische Hervorhebung besonders markiert werden. Zum anderen verweisen Neftalis intralinguale Varianten, die ihm verschiedene (schwedische) soziale Gruppenzugehörigkeiten zuschreiben und gleichzeitig eine schwedische Normsprache hinterfragen, auf eine hohe Sprachkompetenz. (Vgl. Poplack 1980, 615)

4 Alle Übersetzungen aus dem Schwedischen sind meine eigenen. 
Nohas akzentfreies Schwedisch in Kombination mit seinen fehlenden Kenntnissen des Tigrinischen, der Sprache seiner eritreischen Familie, positioniert ihn ebenso wie Neftalis hohe Sprachkompetenz in dem schwedischsprachigen Umfeld, von dem er sich zunächst radikal abgrenzen möchte. Explizit auftretendes interlinguales code-switching zwischen Schwedisch und Tigrinisch, welches sowohl Noha als auch die größte Anzahl der Leser vor Verständnisprobleme stellt, betont Nohas Position in dieser schwedischen Gesellschaft weiterhin:

Er tigrinja är usel, säger han. Det är den. Inga protester från oss. Vad är man utan sitt modersmål? Chowacha, vo chowacha. Vet inte vad det betyder. En gliring av hans tonfall att döma. [...] [Han] berättar [...] att han anställdes tack vare farfar. [...] Mannen hade själv blivit förvånad. På den tiden var han känd för att vara en ouala. Den kan jag. Idiot. Odåga, drönare. (Said 2013, 204)

[Euer Tigrinisch ist erbärmlich, sagt er. Das ist es. Keine Proteste von unserer Seite. Was ist man ohne seine Muttersprache? Chowacha, vo chowacha. Weiß nicht, was das bedeutet. Eine Stichelei seinem Tonfall nach zu urteilen. [...] [Er] erzählt [...], dass er dank meines Großvaters eingestellt wurde. [...] Der Mann war selbst erstaunt gewesen. Zu der Zeit war er dafür bekannt, ein ouala zu sein. Das kenne ich. Idiot. Taugenichts, Faulpelz.]

Während hier folglich Inklusion durch sprachliche Verfremdung vorliegt, zeigt Milfuegos' Tankar mellan hjärtslag Inklusion durch einen verringerten Grad an einer solchen Verfremdung auf. Spanische Ausdrücke werden hier nämlich regelmäßig übersetzt oder erklärt, wodurch Neftalis Sprachstil und - siehe mein Verweis auf Agha und Bauman - Neftali selbst den Rezipienten nicht als ,der fremde Andere‘ erscheint.

\section{Literarische Mehrsprachigkeit als Verfahren der Inklusion}

Der vorhergehende Einblick in die Effekte und Funktionen der Verhandlung von Mehrsprachigkeit in den beiden Romanen Tankar mellan hjärtslag und Väldigt sällan fin hat anhand der Beachtung von literarischen Sprachstilen gezeigt, wie Aghas Konzept des enregisterment die Inklusion in und Exklusion aus sozialen Gruppen steuert. Die Hauptfiguren Neftali und Noha schreiben sich sowohl durch ihre Sprachwahlen als auch durch Kommentare zu diesen eine schwedisch(sprachig)e Zugehörigkeit zu. Diese kann dabei durchaus neben weiteren Zugehörigkeitsempfindungen existieren. Zusätzlich zu den Eigenzuschreibungen wird Neftali und Noha aufgrund der angeführten Effekte der spezifischen Verwendung 
von Mehrsprachigkeit auch von Seiten der schwedischsprachigen Rezipienten diese Inklusion zugesprochen.

Gleichzeitig werden die Romane zu einer Widerstandshandlung gegen soziale Kategorisierungen, indem sie das von Jonsson beschriebene politische Potenzial nutzen, welches Mehrsprachigkeit innewohnt. Allerdings bezieht sich dieser Akt nicht allein auf die Sichtbarmachung anderer Sprachgebräuche. Im selben Maße wie die Hauptfiguren in multikulturellen und mehrsprachigen Milieus verortet werden, wird ihnen ihre schwedische Zugehörigkeit anerkannt. Dadurch verwerfen Tankar mellan hjärtslag und Väldigt sällan fin die Vorstellung eines monokulturellen und monolingualen Schwedens. An deren Stelle fordern sie eine tolerante, offene und multikulturelle Gesellschaft, in der einsprachige Normen sowie geographische Einheiten und familiäre Hintergründe keine alleinige Basis für Nationszugehörigkeiten darstellen.

Wie zu Beginn erwähnt, ist dieses keine neue Haltung in der schwedischen Gegenwartsliteratur. Eine ähnliche Verfahrensweise lässt sich in dem ungefähr zehn Jahre zuvor veröffentlichten Debütroman von Jonas Hassen Khemiri mit dem Titel Ett öga rött verfolgen. Auch dieser Roman unterläuft enge sprachliche Normen dadurch, dass er trotz - oder gerade aufgrund - seiner spezifischen Verwendung sprachlicher Varietäten vorgeprägte Lesererwartungen an multikulturell geprägte Identitäten nicht erfüllt. So hat Wolfgang Behschnitt $(2008,40)$ darauf verwiesen, dass der individuell konstruierte Sprachstil der Hauptfigur Halim, der von zahlreichen Rezipienten fälschlicherweise als authentisches Einwandererschwedisch gelesen wurde, sprachbasierte Vorurteile und deren Einfluss auf Ethnizitätszuschreibungen in Frage stellt. ${ }^{5}$ Halim ist nämlich weder der arabischsprachige Einwanderer, als der er gesehen wird, noch spricht er ausschließlich akzentgefärbtes Schwedisch. Stattdessen ist er ein in Schweden geborener Schwede, der, wie so viele andere, multikulturelle Wurzeln hat.

In allen drei Romanen werden die Hauptfiguren nicht nur mit Ausgeschlossenheit aufgrund ihrer familiären Herkunft konfrontiert, sondern sie streben diese auch bewusst an - unter anderem mithilfe der von Agha beschriebenen sprachlichen Register. Die Rezipienten folgen ihnen dabei auf dem Weg zu der Erkenntnis, dass eine solche Abgrenzung von der geographischen und sozialen Umgebung nicht möglich $\mathrm{zu}$ sein scheint und dass sich ihre internationalen Wurzeln mit einer schwedischen Identität kombinieren lassen. Der Effekt, den diese Strategie der Fokalisierung auf die Leserschaft hat, ist eindeutig: Auch sie gelangt zu der Erkenntnis, dass die Grenzen zwischen homogenen und heterogenen Sprach- und Nationalkonzepten eine Illusion sind.

5 Vgl. hierzu auch Nilsson 2013, insbesondere 51-52. 
Eine Entwicklungslinie in der literarischen Verhandlung multilingualer Identitäten und ihrer selbstverständlichen Position in einer globalen Welt lässt sich dennoch feststellen. In Tankar mellan hjärtslag und Väldigt sällan fin sind es nicht mehr die einwandergeprägten und ausgegrenzten Vororte, die das geographische und soziale Umfeld der Erzählungen bilden, sondern innerstädtische und studentische Lebenswelten. Rein quantitativ betrachtet lässt sich Halims fingiertes ,Einwandererschwedisch` als Hauptsprache von Ett öga rött definieren, während andere Nationalsprachen und Sprachvarietäten in Tankar mellan hjärtslag und Väldigt sällan fin ein geringeres Ausmaß als das sogenannte Standardschwedische einnehmen. Dieses lässt sich als weiteres Indiz für die These deuten, dass die Verwendung von Mehrsprachigkeit in den unterschiedlichen Romanen einer inklusiven Agenda folgt.

Hierzu lässt sich außerdem anführen, dass sich insbesondere Tankar mellan hjärtslag und Ett öga rött durch eine weitere Variante des interkulturellen Dialogs in Form von intertextuellen Bezügen in ein skandinavisches Umfeld einschreiben. Auch wenn es hier nicht um die Beherrschung mehrerer Sprachen oder Sprachvarietäten geht, so lässt sich doch von einer Art Mehrsprachigkeit der Texte sprechen, indem diese Prätexte inkludieren und durch sie kommunizieren. Die inklusiven Funktionen und Effekte der linguistischen Mehrsprachigkeit werden dabei unterstützt, wenn sich Tankar mellan hjärtslag durch Referenzen zu bekannten Autoren wie August Strindberg oder Tomas Tranströmer innerhalb eines schwedischen Literaturkanons positioniert. (Vgl. Milfuegos 2015, 81, 109, 180) Ähnliches gilt für den Verweis auf Henrik Ibsens Peer Gynt als Symbol des Nordischen in Ett öga rött, dessen Figur Ähnlichkeiten zu der Figur von Halim aufweist.

Ebenso wird doch auch der Anspruch der Romane auf eine Erneuerung traditioneller Denkweisen durch intertextuelle Verweise unterstrichen. Statt kanonisierter Texte handelt es sich hierbei um schwedischen Hiphop, ${ }^{6}$ einem Musikstil, der von Vertretern eines high culture-Kanons als low culture klassifiziert wird. Neftalis Identifikation mit diesem Genre, die sich in seinem Schreib- und Sprachstil äußert und durch seine Aussagen gefestigt wird (vgl. Milfuegos 2015, 13), stimmt mit der von Behschnitt beschriebenen Funktion solcher intertextueller Verweise zum Beispiel in Khemiris Ett öga rött überein: Sie „function as symbol of a young, multi-ethnic, and multilingual suburban culture and, at the same time, as a marker of cultural affiliation“ (Behschnitt 2013, 176). Die ungebundene

6 Sowohl Ett öga rött als auch Tankar mellan hjärtslag beziehen sich auf die Band The Latin Kings, Tankar mellan hjärtslag verweist außerdem noch auf die Artisten Mingus und Carlito. (Vgl. Khemiri 2003, 29-30; Milfuegos 2015, 151, 153, 157, 210) 
Kombination kanonisierter und nicht-kanonisierter intertextueller Referenzen entspricht damit den flexiblen Konzepten multilingualer und multikultureller Identitäten, wie sie die Romane postulieren.

\section{Resümee}

Zusammenfassend lässt sich zunächst bestätigen, dass Mehrsprachigkeit und Sprachbewusstsein in der schwedischen Gegenwartsliteratur noch immer eine wesentliche Rolle für die Verhandlung von Identitäten in literarischen Werken spielen, die Fragen nach ethnischen und nationalen Zugehörigkeiten diskutieren. Dabei lassen sich eindeutige Gemeinsamkeiten zwischen den jüngeren Romanen Tankar mellan hjärtslag von Neftali Milfuegos und Väldigt sällan fin von Sami Said und dem älteren ,Prototypen' dieser Art von Literatur, Jonas Hassen Khemiris Ett öga rött, feststellen. Im Vergleich zu Ett öga rött hat sich jedoch der Grad der sprachlichen Exotisierung in den jüngeren Romanen verringert. Diese Abschwächung beruht auf der überwiegend implizit auftretenden Mehrsprachigkeit, der Erläuterung oder der typographischen Integration anderssprachlicher Begriffe sowie der Inklusion von sprachlichen Varietäten. Diese sprachliche Inklusionsarbeit findet vorwiegend auf formalästhetischer Ebene statt, wird aber ebenso auf inhaltlicher Ebene durch Charakter und Verhalten der Figuren reflektiert und unterstützt. Tankar mellan hjärtslag und Väldigt sällan fin weisen dabei unterschiedliche Strategien und Anteile an Mehrsprachigkeit auf. So ist beispielsweise die schwedische Rede des intradiegetisch-autodiegetischen Erzählers Noha akzentfrei, während sie in Neftalis Fall von Soziolekten durchzogen ist. Die Effekte sind doch dieselben: Sprachliche Grenzziehungen werden ebenso aufgehoben wie kulturelle oder geographische Dichotomien, und das ,Fremde“ wird als eine dem ,Eigenen‘ zugehörige Variation entworfen. Durch die inklusiven Verfahren, die sich aus den romanspezifischen Verhandlungen von Mehrsprachigkeit ergeben, werden die Hauptfiguren in dem Entwurf einer multilingualen schwedischen Gesellschaft positioniert, statt aus dieser aufgrund ihrer verschiedenen sprachlichen Fähigkeiten und ihrer unterschiedlichen, familiär bedingten kulturellen Hintergründe ausgeschlossen zu werden. 


\section{Literaturverzeichnis}

Agha, Asif. Language and Social Relations. Cambridge: Cambridge University Press, 2007. Bauman, Richard. „Language, Identity, Performance“. Pragmatics 10.1 (2000): 1-5.

Behschnitt, Wolfgang. „,Willkommen im Vorort': Neue schwedische Literatur zwischen Einwanderer- und Nationalkultur“. Literatur der Migration - Migration der Literatur. Hg. Karin Hoff. Frankfurt: Peter Lang, 2008. 35-48.

Behschnitt, Wolfgang. „The Rhythm of Hip Hop: Multi-Ethnic Slang in Swedish Literature after 2000“. Literature, Language, and Multiculturalism in Scandinavia and the Low Countries. Hg. Wolfgang Behschnitt, Sarah De Mul und Liesbeth Minnaard. Amsterdam und New York: Rodopi, 2013. 175-195.

Eriksson, Harriet, und Saara Haapamäki. „Att analysera litterär flerspråkighet“. Svenskan $i$ Finland 12 (2011): 43-52.

Jonsson, Carla. „Making Silenced Voices Heard. Code-switching in Multilingual Literary Texts in Sweden". Language Mixing and Code-Switching in Writing. Approaches to MixedLanguage Written Discourse. Hg. Mark Sebba, Shahrzad Mahootian und Carla Jonsson. New York: Routledge, 2012. 212-232.

Khemiri, Jonas Hassen. Ett öga rött. Stockholm: Norstedts, 2003.

Laakso, Johanna. „Linguistic approaches to Finno-Ugric literary multilingualism“. Multilingualism and Multiculturalism in Finno-Ugric Literatures. Hg. Johanna Laakso und Johanna Domokos. Münster: LIT, 2011. 26-36.

Landqvist, Hans. „- Kuka ... puhhuu ...? stönade Esaias. Vem pratar?“ Litterär flerspråkighet och språkväxling i Mikael Niemis roman Mannen som dog som en lax. Forschungsberichte vom Institut für Schwedisch. Göteborg: Universität Göteborg, 2012. https://gupea.ub.gu. se/bitstream/2077/30028/3/gupea_2077_30028_3.pdf(30. Juni 2016).

Landqvist, Hans. „,Routtiksi', translated Paul Muotka patiently. ,Kitos.' Mikael Niemi, Meänkieli and Readers Inside and Outside Tornedalen“. Acta Borealia 31.1 (2014): 59-82.

Milfuegos, Neftali. Tankar mellan hjärtslag. Stockholm: Wahlström \& Widstrand, 2015.

Nilsson, Magnus: „Literature in Multicultural and Multilingual Sweden: The Birth and Death of the Immigrant Writer". Literature, Language, and Multiculturalism in Scandinavia and the Low Countries. Hg. Wolfgang Behschnitt, Sarah De Mul und Liesbeth Minnaard. Amsterdam und New York: Rodopi, 2013. 41-61.

Poplack, Shana. „Sometimes l'll start a sentence in Spanish Y TERMINO EN ESPAÑOL: toward a typology of code-switching “. Linguistics 18.7-8 (1980): 581-618.

Said, Sami. Väldigt sällan fin. Stockholm: Natur \& Kultur, 2013.

Hannah Tischmann ist Doktorandin und Lektorin für Literaturwissenschaft an der Abteilung Skandinavistik der Universität Wien. Ihre Forschungsschwerpunkte sind die skandinavischen Gegenwartsliteraturen und die schwedische Arbeiterliteratur. Hier befasst sie sich insbesondere mit den literarischen Verhandlungen gesellschaftspolitischer Fragen wie Multikulturalismus, sozialer Ungleichheit oder Fluchterfahrungen. 